\title{
Circular RNAs in leukemia (Review)
}

\author{
TUĞÇE BALCI OKCANOĞLU1 ${ }^{1}$ and CUMHUR GÜNDÜZ ${ }^{2}$
}

${ }^{1}$ Medical Biology Department, Vocational School of Health Services, Near East University, 99010 Nicosia, North Cyprus; ${ }^{2}$ Department of Medical Biology, Faculty of Medicine, Ege University, 35040 Bornova, Izmir, Turkey

Received September 9, 2018; Accepted November 29, 2018

DOI: $10.3892 /$ br.2018.1178

\begin{abstract}
Circular RNAs (circRNAs) have been demonstrated to be biomarkers in human cancers. CircRNAs are majorly recognized in the transcript formation of eukaryotic genes. Research has also revealed that circRNAs regulate gene expression at the transcriptional, post-transcriptional, and translational levels. Notably, there have been studies demonstrating that they contribute to the improvement of various diseases, including cancer. In this regard, they have potential applications in the diagnosis and treatment of cancer. In circRNA studies of blood fluids, plasma circRNAs have been identified as biomarkers in human cancers. For instance, the acute myeloid leukemia-associated hsa_circ_0004277 has been reported to be a biomarker in targeted treatments. This links with circRNAs being highly expressed in hematopoietic tissue; in haematopoiesis, the cell states are controlled by the main regulators and the complex circuits of the RNA family. In particular, circRNA serve a role in cellular processes including self-renewal, apoptosis and proliferation. In the current review, the aim was to explain the role of the defined pathogenic circRNAs derived from leukemia fusion genes and of hsa_circ_0004277 in leukemia cells.
\end{abstract}

\section{Contents}

1. Introduction

2. Characteristics of circRNAs

3. Biogenesis and function of circRNAs

4. CircRNAs-F-circRNAs in leukemia

5. Hsa_circ_0004277 and leukemia

6. Conclusions and perspectives

Correspondence to: Professor Tuğçe Balcı Okcanoğlu, Medical Biology Department, Vocational School of Health Services, Near East University, Near East Boulevard, 99010 Nicosia, North Cyprus E-mail: tbalcii@gmail.com

Key words: circular RNA, fusion circular RNAs, leukemia, hsa_circ_0004277

\section{Introduction}

Non-coding RNAs (ncRNAs), which comprise long non-coding RNAs (lncRNAs), short microRNAs (miRNA/miRs) and circular RNAs (circRNAs), constitute the majority of total RNAs in the eukaryotic transcriptome $(1,2)$. CircRNAs were first identified in viruses in 1970, and subsequently in eukaryotic cells $(3,4)$. CircRNAs are a relatively large group of RNAs that form stable closed circles. A major proportion of ncRNAs and circRNAs are involved in the regulation of transcriptional and post-transcriptional gene expression (5). CircRNAs are produced from the back-splicing of intronic and/or exonic RNA (2). They serve a significant role in cancer development, metastasis and response to treatment (6). The specificity of circRNA in disease states and the stability of circRNA in body fluids indicate that they may be used molecular markers in the diagnosis of cancer (6-9). A high number of circRNAs have been identified following the development of sequencing and bioinformatics analysis techniques (10-12). In particular, abnormal expressions of circRNAs have been identified in leukemia. For instance, hsa_circ_0035381, hsa_circ_0004136 and hsa_circ_0058058 are reportedly upregulated while hsa circ_0017446 and hsa_circ_0004277 are downregulated in acute myeloid leukemia (6). In the present review, the aim was to explain the role of defined pathogenic circRNAs derived from leukemia fusion genes [mixed lineage leukemia-ALL1 fused gene from chromosome 9 (MLL-AF9) and promyelocytic leukemia-retinoic acid receptor $\alpha$ (PML-RARA)] and hsa_circ_0004277 in leukemia cells.

\section{Characteristics of circRNAs}

CircRNAs are highly diverse, originating from any region of the genomic subsequences (exon-intron circRNAs, intronic circRNAs, exonic circRNAs) (2,12-16). The majority of the circRNAs are generated from one or more excess exons (17-20). The exonic circRNAs predominantly reside in the cytoplasm $(21,22)$. They have a more stable structure than linear RNAs, with 5'-3' polarities and no polyadenylation tails (10). CircRNAs can be degraded by RNA exonuclease or ribonuclease (RNase) R (23). CircRNAs are enriched in exosomes (24).

It is established that circRNAs are relatively common in eukaryotic transcriptomics and are stable in intracellularly in the cytoplasm and in the blood (7). CircRNAs can be secreted into body fluids or developed in exosomes, and as such have 
emerged as major biomarkers for cancer diagnosis $(8,24,25)$. It has been demonstrated that the expression of circRNA is specific, and that the molecules serve as miRNA sponges to regulate gene expression (5,26-28). CircRNAs may have biological activities by acting as miRNA sponges and/or by binding with RNA-binding proteins (RBPs) and translation peptides $(5,24,29,30)$. In the study of cancer, an ongoing aim is to identify potential biomarkers that are differentially expressed between healthy and cancerous tissues. In this regard, there are studies indicating that circRNAs are associated with the initiation and development of cancer (31-33).

\section{Biogenesis and function of circRNAs}

CircRNAs are divided into three groups: Exonic circRNAs, intronic circRNAs and exon-intron circRNAs. Throughout the formation of all types of circRNAs, characteristic sequences are preserved. Sequences from the downstream and upstream regions enable the formation of mature circRNAs followingback-splicing by covalently linking in the reverse direction (34). While the effect of back-splicing is less significant for linear RNAs due to their durability and half-life, circRNAs are present in excess in cells (35). The first back-splicing mechanism works due to complementary intron matches that serve a role in the formation of exonic circRNA (17). CircRNA may interact with other RNA molecules and DNA, including mRNA and lncRNA $(36,37)$.

CircRNAs are associated with various diseases, including cancer in particular (31,38-40). It is established that there is an association between circRNA and miRNA in various cancers (30,31). CircRNAs act as miRNA sponges, RBP sponges and transcription and translation regulators. CircRNAs have important functions in the regulation of gene expression $(5,14,41,42)$. Reportedly there is deregulation of the splicing mechanisms in acute myeloid leukemia (AML), leading to abnormalities in the expressed circRNAs in leukemia cells; in turn, the altered circRNAs can have functions in leukemogenesis (6).

It has been shown that circRNAs can bind to miRNA as RNA sponges, and by influencing miRNA activities, may increase gene expression and contribute to the development of tumours (5,43-45). In particular, it has been reported that circRNAs can increase or suppress the development of cancer genes associated with migration, differentiation, proliferation and carcinogenesis by suppressing miRNA species (46). For instance, among the first circRNAs to be identified, CiRS-7, contains an miR-7 binding site and acts as an miR-7 sponge, thus reducing the effect of miR-7 on its target mRNAs $(47,48)$. Overexpression of ciRS-7 in colorectal cancer cells has been reported to eliminate the tumor suppressor function of miR-7 (49).Circ-ZNF609 is also a cancer-associated gene, being observed to inhibit proliferation in colorectal cancer cells (50).

\section{CircRNAs-F-circRNAs in leukemia}

CircRNAs have been studied in hematopoietic malignancies in primary patient samples. In bone marrow from patients, Salzman et al (10) determined that hyperdiploid B-linage acute lymphoblastic leukemia (ALL) in childhood could rearrange chromosomal regions using an RNA-seq method. Furthermore, they conducted research on the rearrangement in specialized genomic exon regions subject to RNA splicing. In the ALL patients, they identified hundreds of genes that could produce circRNAs. Fusion genes encoded by abnormal chromosomal translocations have been demonstrated to be associated with malignant haematological diseases. Chromosomal rearrangements in tumour cells affect ncRNA levels through the formation of fusion-circRNAs (f-circRNAs) (51).

There are inherent chromosomal translocations in various types of leukemia. A recurrent translocation is PML-RARA in patients with acute promyelocytic leukemia (APL) (52). Recently, studies on circRNA expression in AML have been conducted. Guarnerio et al (6) demonstrated that circRNAs are derived from the transcription of fusion genes that occur from chromosomal translocations. Indeed in various studies, circRNAs have been associated with many cancers, including AML with MLL-AF9 and promyelocytic leukemia with PML-RARA. Many are established as f-circRNAs. Abnormal expression of circRNAs may be the result of distant interrelated introns and introns of translational genes that contain repeating sequences complementary to each other. Novel back-splicing events are supposed to support their formation into abnormal circRNAs (53).

A previous review noted that cancer-associated fusion genes may cause the expression of f-circRNAs, including the pro-proliferative, proto-oncogenic f-circPR and f-circM9_1 (6). You and Conrad (54) determined differently expressed f-circRNAs in APL and AML. Such is in accordance with f-circRNAs being established to serve an active role in haematological events (53). Li et al (53) reported that hsa_circ_0004277 in AML patients was downregulated.

Circ-HIPK2 has been indicated to serve a role as a transcription coactivator in nuclear bodies and is considered to have important functions in the formation and development of AML $(55,56)$. Li et al (57) demonstrated that expression of circ-HIPK2 affected ATRA-induced differentiation of APL cells. In addition, the expression of circ-HIPK2 was lower in AML cells compared with APL cells, and overexpression of circ-HIPK2 increased differentiation in NB4 cells (APL cells with PML-RARA). Therefore, HIPK2 appeared to be required for differentiation of ATRA-induced APL cells. This suggests that circ-HIPK2 may be a biomarker in APL cells.

There are many chromosomal translocations in several types of leukemia. These chromosomal translocations may occur due to the instability of the tumour cells' genome. The abnormal chromosomal translocations leading to the rearrangement of non-homologous chromosomes brings two separate genes together, giving rise to the production of a fusion genome. Chromosomal translocations can encode such oncogenic fusion proteins in tumor formation, these proteins may be a cause of cancer (58).

While fusion of circular RNA (f-circRNA) occurs, gene fusions can also cause defects in mRNA. For example, PML-RARA and MLL-AF9 genes are fused, and thus form f-circM9 and f-circPR. Knockout of f-circM9 and f-circPR can increase apoptosis in cancer cells and increase drug sensitivity to agents such as arsenic (6). These data indicate that f-circM9 and f-circPR have a role in haematological malignancies (Fig. 1). Guarnerio et al (6) documented that expression of f-circRNAs (f-circPR and f-circM9) in leukemia increased 


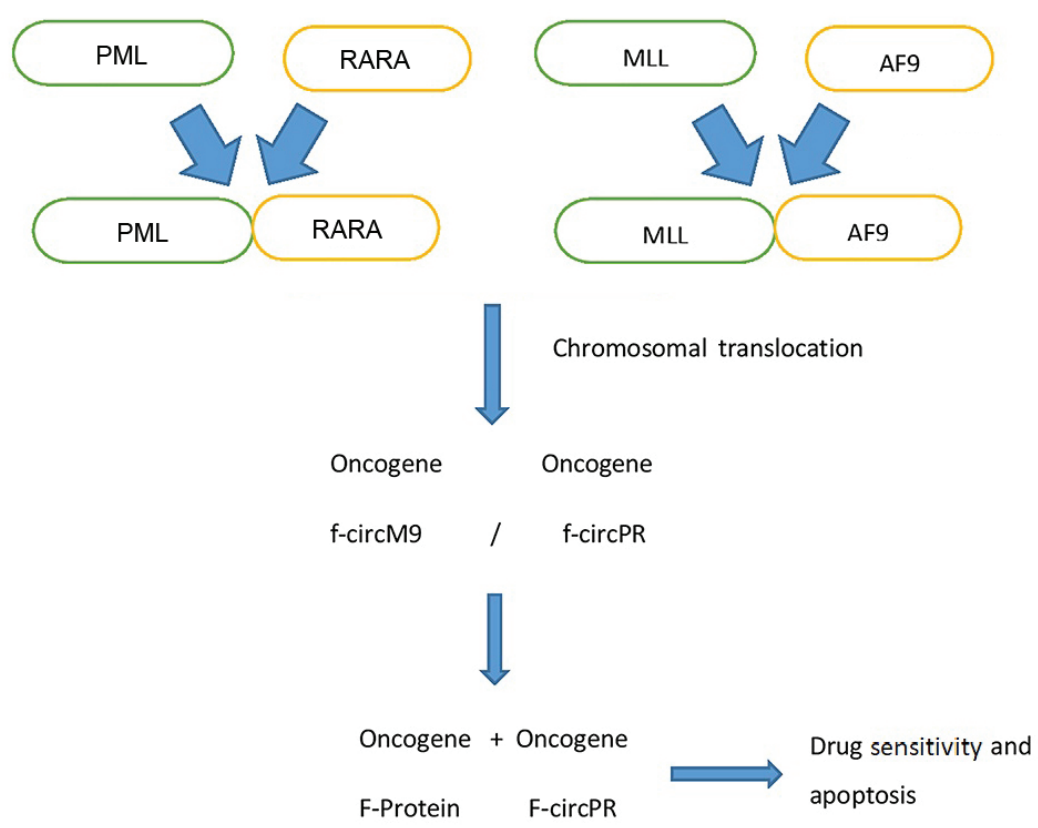

Figure 1. Roles of the PML-RARA and MLL/AF9 fusion genes in the apoptosis and drug of sensitivity of leukemia cells. PML-RARA and MLL-AF9 genes become fused, and f-circM9 and f-circPR are formed. Knockout of f-circM9 and f-circPR may increase apoptosis and drug sensitivity. PML-RARA, promyelocytic leukemia-retinoic acid receptor $\alpha$; MLL-AF9, mixed lineage leukemia-ALL1 fused gene from chromosome 9; F- fusion; circ, circular RNA.

cell proliferation and clonogenicity. They also showed that the phenotype of cells with silenced f-circRNA was reversed. Thus, these f-circRNAs may have pro-proliferative and proto-oncogenic properties in leukemia. Indeed, it has been documented that f-circRNAs (produced from PML-RARA and MLL-AF9 fusion genes) have a role in the survival of leukemia cells (6).

The f-circRNAs expressed in human leukemia cells have been associated with disease formation (59). However, the f-circRNA does not cause leukemia alone. Combined expression of F-circM9 and MLL-AF9 fusion protein have been reported to increase proliferation; notably only cells expressing the fusion protein exhibited increased colonies. The expression of f-circRNAs may provide protection for tumour cells in standard leukemia therapy, including against arsenic trioxide, and may also provide survival benefit to leukemic cells during standard leukemia treatment with cytarabine (59).

\section{Hsa_circ_0004277 and leukemia}

Hsa_circ_0004277 is located in the region of chromosome 10: $1125950-1126416$. WDR37 is the gene symbol of hsa circ_0004277 (60). WDR37, located on the same chromosome, is the linear isoform of Hsa_circ_0004277 (53). This family is associated with apoptosis, signalling pathways and cellular events including the cell cycle (60). Wei et al (53) identified hsa_circ_0004277 as a target and biomarker in the treatment of AML. When the circRNA-miRNA-mRNA interaction for hsa_circ_0004277 has been examined by bioinformatics analysis, it has provided insight into the underlying mechanism of its function in AML. For instance, while hsa_circ_0035381, hsa_circ_0004136 and hsa_circ_0058058 are upregulated, the hsa_circ_0017446 and hsa_circ_0004277 are downregulated in AML (53). Newly diagnosed cases of AML have been found to exhibit downregulated hsa_circ_0004277, while, the expression of hsa_circ_0004277 was increased in complete remission cases, and the level once again downregulated in relapsed/refractory cases. These results indicate that hsa_circ_0004277 may be used as a diagnostic marker and therapeutic target in AML (53).

\section{Conclusions and perspectives}

In the current review, we sought to summarize the formation of circRNAs and their characteristics and functional properties, and the roles of the defined pathogenic circRNAs derived from the leukemia fusion genes and hsa_circ_0004277 in leukemia cells. Studies on circRNAs for clinical diagnosis and treatment are viewed as a guide for translational and clinical medical developments. Considering the functions and mechanisms of circRNAs, they may be considered among the main topics of cancer research. Research on these molecules may provide insight into the fundamental molecular events involved. The use of circRNAs as diagnostic biomarkers in cases of cancer metastasis may be clinically significant. Different cirRNA expression profiles correlate with clinical features, including tumor stage and recurrence of metastasis, supported by recent RNA-seq studies $(61,62)$. Additionally, circRNAs are established as being markedly more stable than linear RNAs.

Different expression profiles of circRNAs have been identified in studies on tissues and blood. The expression of circRNAs is different from that of miRNAs and lncRNAs. By combining with various biomarkers, it is possible to model for prognosis and increase the accuracy and specificity of diagnosis $(63,64)$. The consequences of molecular circuits that control cellular differentiation in the hematopoietic system are a topic of study. In haematopoiesis, differentiated cell states can be controlled by transcriptional circuits linked to each other (65). Recent study of f-circRNAs has revealed the oncogenic roles of abnormal circRNAs in leukemogenesis (51). Studies have also shown that 
circRNAs may be molecular markers in tumours and affect cell death.

CircRNAs have potential in targeted cancer therapy, in that they may be used as a sponge for binding to abnormally expressed regulatory RNAs and proteins (e.g., RBPs), to thus reduce oncogenic activities (66). For instance, certain f-circRNAs have been found to be resistant to chemotherapy in leukemia patients. The knocking out of f-circM9 expression in leukemia cells can lead to apoptosis. In another example, while hsa_circ_0004277 showed low expression in newly diagnosed AML patients, no difference was determined in patients following treatment. In patients with recurrent disease, hsa_circ_0004277 has been observed to be downregulated (6). For this reason, hsa_circ_0004277 may be a diagnostic marker in AML. The overall stability of the circRNAs may be useful in indicating diseases that can be identified by body fluids.

In conclusion, considering the functions and mechanisms of circRNAs, they may be among the main topics in cancer research. In particular, circRNAs may be a diagnostic marker for leukemia. An improved understanding of circRNA biology may provide a guide for novel therapeutic targets.

\section{Acknowledgements}

Not applicable.

\section{Funding}

Not applicable.

\section{Availability of data and materials}

Not applicable.

\section{Authors' contributions}

TBO and CG searched the related literature. TBO wrote the manuscript. All authors discussed the content and contributed to the final manuscript.

\section{Ethics approval and consent to participate}

Not applicable.

\section{Consent for publication}

Not applicable.

\section{Competing interests}

The authors declare that they have no competing interests.

\section{References}

1. Warner JR: The economics of ribosome biosynthesis in yeast. Trends Biochem Sci 24: 437-440, 1999.

2. Jeck WR, Sorrentino JA, Wang K, Slevin MK, Burd CE, LiuJ, et al: Circular RNAs are abundant, conserved, and associated with ALU repeats. RNA 19: 141-157, 2013.

3. Sanger HL, Klotz G, Riesner D, Gross HJ and Kleinschmidt AK Viroids are single-stranded covalently closed circular RNA molecules existing as highly base-paired rod-like structures. Proc Natl Acad Sci USA 73: 3852-3856, 1976.
4. Hsu MT and Coca-Prados M: Electron microscopic evidence for the circular form of RNA in the cytoplasm of eukaryotic cells. Nature 280: 339-340, 1979.

5. Hansen TB, Jensen TI, Clausen BH, Bramsen JB, Finsen B, Damgaard CK and Kjems J: Natural RNA circles function as efficient microRNA sponges. Nature 495: 384-388, 2013.

6. Guarnerio J, Bezzi M, Jeong JC, Paffenholz SV, Berry K, Naldini MM, Lo-Coco F, Tay Y, Beck AH and Pandolfi PP: Oncogenic role of fusion-circRNAs derived from cancer-associated chromosomal translocations. Cell 165: 289-302, 2016.

7. Memczak S, Papavasileiou P, Peters O and Rajewsky N: Identification and characterization of circular RNAs as a new class of putative biomarkers in human blood. PLoS One 10: e0141214, 2015.

8. Bahn JH, Zhang Q, Li F, Chan TM, Lin X, Kim Y, Wong DT and Xiao X: The landscape of microRNA, Piwi-interacting RNA, and circular RNA in human saliva. Clin Chem 61: 221-230, 2015.

9. Szabo L, Morey R, Palpant NJ, Wang PL, Afari N, Jiang C, Parast MM, Murry CE, Laurent LC and Salzman J: Statistically based splicing detection reveals neural enrichment and tissue-specific induction of circular RNA during human fetal development. Genome Biol 16: 126, 2015.

10. Salzman J, Gawad C, Wang PL, Lacayo N and Brown PO: Circular RNAs are the predominant transcript isoform from hundreds of human genes in diverse cell types. PLoS One 7: e30733, 2012.

11. Guo JU, Agarwal V, Guo H and Bartel DP: Expanded identification and characterization of mammalian circular RNAs. Genome Biol 15: 409, 2014

12. Memczak S, Jens M, Elefsinioti A, Torti F, Krueger J, Rybak A, Maier L, Mackowiak SD, Gregersen LH, Munschauer M, et al: Circular RNAs are a large class of animal RNAs with regulatory potency. Nature 495: 333-338, 2013.

13. Zhang Y, Zhang XO, Chen T, Xiang JF, Yin QF, Xing YH, Zhu S, Yang L and Chen LL: Circular intronic long noncoding RNAs. Mol Cell 51: 792-806, 2013.

14. Li Z, Huang C, Bao C, Chen L, Lin M, Wang X, Zhong G, Yu B, $\mathrm{Hu}$ W, Dai L, et al: Exon-intron circular RNAs regulate transcription in the nucleus. Nat Struct Mol Biol 22: 256-264, 2015.

15. Shen $\mathrm{T}$, Han $\mathrm{M}$, Wei $\mathrm{G}$ and $\mathrm{Ni} \mathrm{T}$ : An intriguing RNA species - perspectives of circularized RNA. Protein Cell 6: 871-880, 2015.

16. Chen LL and Yang L: Regulation of circRNA biogenesis. RNA Biol 12: 381-388, 2015.

17. Zhang XO, Wang HB, Zhang Y, Lu X, Chen LL and Yang L: Complementary sequence-mediated exon circularization. Cell 159: 134-147, 2014.

18. Chen I, Chen CY and Chuang TJ: Biogenesis, identification, and function of exonic circular RNAs. Wiley Interdiscip Rev RNA 6: 563-579, 2015.

19. Starke S, Jost I, Rossbach O, Schneider T, Schreiner S, Hung LH and Bindereif A: Exon circularization requires canonical splice signals. Cell Reports 10: 103-111, 2015.

20. Suzuki H, Aoki Y, Kameyama T, Saito T, Masuda S, Tanihata J, Nagata T, Mayeda A, Takeda S and Tsukahara T: Endogenous multiple exon skipping and back-splicing at the DMD mutation hotspot. Int J Mol Sci 17: E1722, 2016.

21. Salzman J, Chen RE, Olsen MN, Wang PL and Brown PO: Cell-type specific features of circular RNA expression. PLoS Genet 9: e1003777, 2013.

22. Westholm JO, Miura P, Olson S, Shenker S, Joseph B, Sanfilippo P, Celniker SE, Graveley BR and Lai EC: Genome-wide analysis of drosophila circular RNAs reveals their structural and sequence properties and age-dependent neural accumulation. Cell Reports 9: 1966-1980, 2014.

23. Suzuki H, Zuo Y, Wang J, Zhang MQ, Malhotra A and Mayeda A: Characterization of RNase R-digested cellular RNA source that consists of lariat and circular RNAs from pre-mRNA splicing. Nucleic Acids Res 34: e63, 2006.

24. Li Y, Zheng Q, Bao C, Li S, Guo W, Zhao J, Chen D, Gu J, He X and Huang S: Circular RNA is enriched and stable in exosomes: A promising biomarker for cancer diagnosis. Cell Res 25: 981-984, 2015.

25. Galasso M, Costantino G, Pasquali L, Minotti L, Baldassari F, Corrà F, Agnoletto $C$ and Volinia S: Profiling of the predicted circular RNAs in ductal in situ and invasive breast cancer: A pilot study. Int J Genomics 2016: 4503840, 2016.

26. Wang PL, Bao Y, Yee MC, Barrett SP, Hogan GJ, Olsen MN, Dinneny JR, Brown PO and Salzman J: Circular RNA is expressed across the eukaryotic tree of life. PLoS One 9: e90859, 2014. (CrossRef) (PubMed). 
27. Rybak-Wolf A, Stottmeister C, Glažar P, Jens M, Pino N, Giusti S, Hanan M, Behm M, Bartok O, Ashwal-Fluss R, et al: Circular RNAs in the mammalian brain are highly abundant, conserved, and dynamically expressed. Mol Cell 58: 870-885, 2015. (CrossRef) (PubMed).

28. Zheng Q, Bao C, Guo W, Li S, Chen J, Chen B, Luo Y, Lyu D, $\mathrm{Li} \mathrm{Y}$, Shi G, et al: Circular RNA profiling reveals an abundant circHIPK3 that regulates cell growth by sponging multiple miRNAs. Nat Commun 7: 11215, 2016.

29. Granados-Riveron JT and Aquino-Jarquin G: The complexity of the translation ability of circRNAs. Biochim Biophys Acta 1859 $1245-1251,2016$

30. Wang Y and Wang Z: Efficient backsplicing produces translatable circular mRNAs. RNA 21: 172-179, 2015

31. Dong Y, He D, Peng Z, Peng W, Shi W, Wang J, Li B, Zhang C and Duan C: Circular RNAs in cancer: An emerging key player. J Hematol Oncol 10: 2, 2017.

32. Qu S, Zhong Y, Shang R, Zhang X, Song W, Kjems J and Li H: The emerging landscape of circular RNA in life processes. RNA Biol 14: 992-999, 2017.

33. Xin Z, Ma Q, Ren S, Wang G and Li F: The understanding of circular RNAs as special triggers in carcinogenesis. Brief Funct Genomics 16: 80-86, 2017.

34. Vicens Q and Westhof E: Biogenesis of Circular RNAs. Cell 159: 13-14, 2014.

35. Qu S, Yang X, Li X, Wang J, Gao Y, Shang R, Sun W, Dou K and Li H: Circular RNA: A new star of noncoding RNAs. Cancer Lett 365: 141-148, 2015.

36. Chen CY and Sarnow P: Initiation of protein synthesis by the eukaryotic translational apparatus on circular RNAs. Science 268: 415-417, 1995.

37. Ashwal-Fluss R, Meyer M, Pamudurti NR, Ivanov A, Bartok O, Hanan M, Evantal N, Memczak S, Rajewsky N and Kadener S: circRNA biogenesis competes with pre-mRNA splicing. Mo Cell 56: 55-66, 2014.

38. Rinn JL and Chang HY: Genome regulation by long noncoding RNAs. Annu Rev Biochem 81: 145-166, 2012.

39. Mercer TR, Dinger ME and Mattick JS: Long non-coding RNAs: Insights into functions. Nat Rev Genet 10: 155-159, 2009.

40. Perriman R and Ares M Jr: Circular mRNA can direct translation of extremely long repeating-sequence proteins in vivo. RNA 4: 1047-1054, 1998.

41. Zhang XO, Dong R, Zhang Y, Zhang JL, Luo Z, Zhang J, Chen LL and Yang L: Diverse alternative back-splicing and alternative splicing landscape of circular RNAs. Genome Res 26: 1277-1287, 2016.

42. Hentze MW and Preiss T: Circular RNAs: Splicing's enigma variations. EMBO J 32: 923-925, 2013.

43. Ebert MS and Sharp PA: MicroRNA sponges: Progress and possibilities. RNA 16: 2043-2050,2010.

44. Ebert MS and Sharp PA: Emerging roles for natural microRNA sponges. Curr Biol 20: R858-R861, 2010.

45. Chen LL: The biogenesis and emerging roles of circular RNAs. Nat Rev Mol Cell Biol 17: 205-211, 2016.

46. Conn SJ, Pillman KA, Toubia J, Conn VM, Salmanidis M, Phillips CA, Roslan S, Schreiber AW, Gregory PA and Goodall GJ: The RNA binding protein quaking regulates formation of circRNAs. Cell 160: 1125-1134, 2015.

47. Hansen TB, Kjems J and Damgaard CK: Circular RNA and miR-7 in cancer. Cancer Res 73: 5609-5612, 2013.

48. Junn E, Lee KW, Jeong BS, Chan TW, Im JY and Mouradian MM Repression of alpha-synuclein expression and toxicity by microRNA-7. Proc Natl Acad Sci USA 106: 13052-13057, 2009.

49. Weng W, Wei Q, Toden S, Yoshida K, Nagasaka T, Fujiwara T, Cai S, et al: Circular RNA ciRS-7-A Promising Prognostic Biomarker and a Potential Therapeutic Target in Colorectal Cancer. Clin Cancer Res 23: 3918-3928, 2017.
50. Wu L, Xia J, Yang J, Shi Y, Xia H, Xiang X, et al: Circ-ZNF609 promotes migration of colorectal cancer by inhibiting Glil expression via microRNA-150. J BUON 23: 1343-1349, 2018.

51. Bonizzato A, Gaffo E, Te Kronnie G and Bortoluzzi S: CircRNAs in hematopoiesis and hematological malignancies. Blood Cancer J 6: e483, 2016

52. Dos Santos GA, Kats L and Pandolfi PP: Synergy against PML-RARa: Targeting transcription, proteolysis, differentiation, and self-renewal in acute promyelocytic leukemia. J Exp Med 210: 2793-2802, 2013.

53. Li W, Zhong C, Jiao J, Li P, Cui B, Ji C and Ma D: Characterization of hsa_circ_0004277 as a new biomarker for acute myeloid leukemia via circular RNA profile and bioinformatics analysis. Int J Mol Sci 18: 597, 2017.

54. You X and Conrad TO: Acfs: Accurate circRNA identification and quantification from RNA-Seq data. Sci Rep 6: 38820, 2016.

55. Li XL, Arai Y, Harada H, Shima Y, Yoshida H, Rokudai S, Aikawa Y, Kimura A and Kitabayashi I: Mutations of the HIPK2 gene in acute myeloid leukemia and myelodysplastic syndrome impair AML1- and p53-mediated transcription. Oncogene 26: 7231-7239, 2007.

56. Shima Y, Honma Y and Kitabayashi I: PML-RAR $\alpha$ and its phosphorylation regulate pml oligomerization and HIPK2 stability. Cancer Res 73: 4278-4288, 2013.

57. Li S, Ma Y, Tan Y, Ma X, Zhao M, Chen B, Zhang R, Chen Z and Wang K: Profiling and functional analysis of circular RNAs in acute promyelocytic leukemia and their dynamic regulation during all-trans retinoic acid treatment. Cell Death Dis 9: 651, 2018.

58. Greuber EK, Smith-Pearson P, Wang J and Pendergast AM: Role of ABL family kinases in cancer: From leukaemia to solid tumours. Nat Rev Cancer 13: 559-571, 2013.

59. Greene J, Baird AM, Brady L, Lim M, Gray SG, McDermott R and Finn SP: Circular RNAs: Biogenesis, Function and Role in Human Diseases, Front Mol Biosci 4: 38, 2017.

60. http://www.circbase.org/cgi-bin/singlerecord.cgi?id=hsa circ_0004277

61. Xu L, Zhang M, Zheng X, Yi P, Lan $\mathrm{C}$ and $\mathrm{Xu} \mathrm{M}$ : The circular RNA ciRS-7 (Cdrlas) acts as a risk factor of hepatic microvascular invasion in hepatocellular carcinoma. J Cancer Res Clin Oncol 143: 17-27, 2017.

62. Zhang Y, Li J, Yu J, Liu H, Shen Z, Ye G, Mou T, Qi X and Li G: Circular RNAs signature predicts the early recurrence of stage III gastric cancer after radical surgery. Oncotarget 8: 22936-22943, 2017.

63. Gong Z, Zhang S, Zhang W, Huang H, Li Q, Deng H, Ma J, Zhou M, Xiang J, Wu M, et al: Long non-coding RNAs in cancer. Sci China Life Sci 55: 1120-1124, 2012.

64. Huang M,Zhong Z,Lv M,Shu J,Tian Q and Chen J: Comprehensive analysis of differentially expressed profiles of lncRNAs and circRNAs with associated co-expression and ceRNA networks in bladder carcinoma. Oncotarget 7: 47186-47200, 2016.

65. Novershtern N, Subramanian A, Lawton LN, Mak RH, Haining WN, McConkey ME, Habib N, Yosef N, Chang CY, Shay T, et al: Densely interconnected transcriptional circuits control cell states in human hematopoiesis. Cell 144: 296-309, 2011.

66. Bachmayr-Heyda A, Reiner AT, Auer K, Sukhbaatar N, Aust S, Bachleitner-Hofmann T, Mesteri I, Grunt TW, Zeillinger R and Pils D: Correlation of circular RNA abundance with proliferation - exemplified with colorectal and ovarian cancer, idiopathic lung fibrosis, and normal human tissues. Sci Rep 5: $8057,2015$. 